\title{
Matrix Reordering Analyzer: Integration with R Software and improvements in support for running experiments.
}

\section{Matheus Peron Baroni, Celmar Guimaraes Da Silva.}

\begin{abstract}
The project aims to continue the development of the Matrix Reordering Analyser (MRA) by enhancing internal processes of experiment execution and the integration with $\mathrm{R}$ Software, in which several seriation algorithms already available on its libraries could be used as matrix reordering algorithms by the MRA.
\end{abstract}

\section{Key words:}

Information visualization, reorderable matrices, seriation.

\section{Introduction}

Reordering matrices are a data structure underlying different tabular interactive graphs, such as heat maps.

The Matrix Reordering Analyzer ${ }^{1}$ (MRA) tool (Image 1) has a set of algorithms for matrix reordering and the ability to compare them through the runtime and quality of the generated matrix as reordering output.

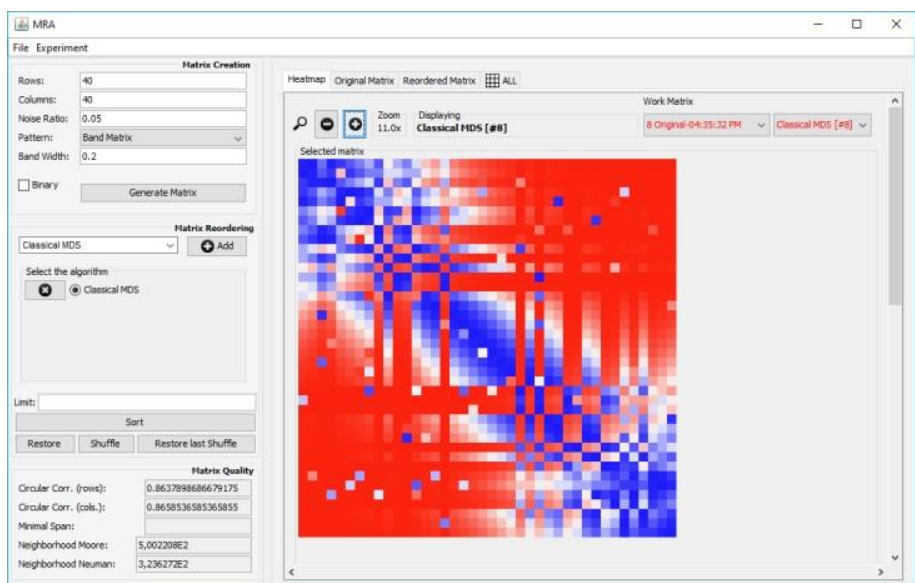

Image 1. MRA interface.

In this context, this work aims to provide continuity to the development of the tool in two aspects. First, it concerns its integration with $\mathrm{R}$ software, in which several seriation algorithms already available on its libraries can be used as matrix reordering algorithms by the MRA. The second objective indicates the need to improve aspects of the internal processes of experiment execution, especially the handle of previously used sets of input matrices, admitting an organized storage of experiments data already executed and facilitating further references and data complementation.

\section{Results and Discussion}

Since MRA is a software developed in Java, it was possible to integrate with $\mathrm{R}$ through the Java/ $R$ Integration (JRI) interface from the rJava library². JRI admits command execution and storage variables of $R$ by Java.

With this interface, it was inserted the reordering methods of the Seriation ${ }^{3}$ and Corrplot ${ }^{4}$ libraries, totalling 41 methods, thus tripling the reordering methods amount available in the MRA.
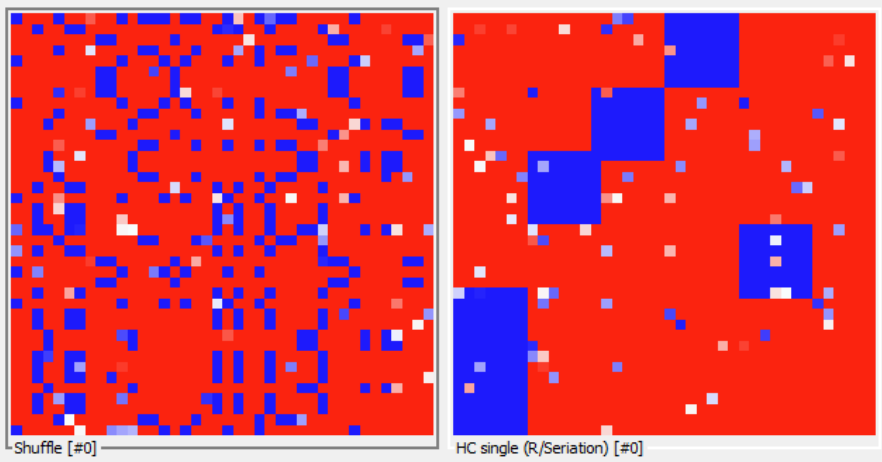

Image 2. left: matrix used as input; right: input matrix reordered in the MRA using the method "HC single" of R's Seriation library.

A second stage of the work was foreseen, where it was proposed improvements in the support to the execution of experiments. This, due to the reduced period of my initiation (six months), unfortunately, could not be concluded.

\section{Conclusions}

The integration of reordering algorithms from $R$ to the MRA will be of great value for the execution of experiments that compare quality and runtime between them, enabling the identification of the most appropriate methods for each dataset and the comparison of algorithms that were already present in the tool with the equivalent ones from the $R$ libraries.

\section{Acknowledgement}

To SAE and PRP for sponsoring my research, and Prof. Celmar Guimarães da Silva for the support and opportunity to do my first academic research.

1 SILVA, C. G. da et al. PQR sort: using PQR trees for binary matrix reorganization. Journal of the Brazilian Computer Society, v. 20, n. 1, 2014.

2 URBANEK, S. How to talk to strangers: ways to leverage connectivity between R, Java and Objective C. Computational Statistics, v. 24, n. 2, p. $303-$ 311,15 nov. 2008

${ }^{3}$ HAHSLER, M.; HORNIK, K.; BUCHTA, C. Getting Things in Order: An Introduction to the R Package seriation. Journal of Statistical Software, v. 25, n. 3, 2008.

${ }^{4}$ WEI, T. et al. Package 'corrplot'. Statistician, v. 56, p. 316-324, 2017. 